\title{
An Advance-Tracing Boundary Element Method in the Time Domain for Solving the Radiating Problem of an Arbitrary Obstacle Accelerating Randomly
}

\author{
Jui-Hsiang Kao \\ Department of Systems Engineering and Naval Architecture, National Taiwan Ocean University, Keelung, Taiwan \\ Correspondence should be addressed to Jui-Hsiang Kao; jhkao@mail.ntou.edu.tw
}

Received 9 February 2017; Revised 2 April 2017; Accepted 27 April 2017; Published 4 June 2017

Academic Editor: Peter Dabnichki

Copyright (C) 2017 Jui-Hsiang Kao. This is an open access article distributed under the Creative Commons Attribution License, which permits unrestricted use, distribution, and reproduction in any medium, provided the original work is properly cited.

\begin{abstract}
This research develops an Advance-Tracing Boundary Element Method in the time domain to calculate the waves that radiate from an immersed obstacle moving with random acceleration. The moving velocity of the immersed obstacle is multifrequency and is projected along the normal direction of every element on the obstacle. The projected normal velocity of every element is presented by the Fourier series and includes the advance-tracing time, which is equal to a quarter period of the moving velocity. The moving velocity is treated as a known boundary condition. The computing scheme is based on the boundary integral equation in the time domain, and the approach process is carried forward in a loop from the first time step to the last. At each time step, the radiated pressure on each element is updated until obtaining a convergent result. The Advance-Tracing Boundary Element Method is suitable for calculating the radiating problem from an arbitrary obstacle moving with random acceleration in the time domain and can be widely applied to the shape design of an immersed obstacle in order to attain security and confidentiality.
\end{abstract}

\section{Introduction}

The radiated wave from any immersed obstacle moving with acceleration is detected for various purposes, such as remote sensing and military defense, but the literature rarely discusses this kind of radiated wave. However, this issue is important for a well-shaped immersed obstacle, in order for it to attain security and confidentiality.

Radiation from a vibrating obstacle in the frequency domain has been widely investigated since the 1870s. According to Rayleigh [1], the radiation from a vibrating plate is presented by the Rayleigh integral in the frequency domain. Kropp and Svensson [2] used the time-domain formulations of equivalent sources to approximate Green's functions to simplify the radiation problem. Ochmann [3] developed the source simulation technique (SST) in the frequency domain for faster computation of complex structures. Wu [4] applied the direct boundary element method in computing the radiation from a regular thin body. Sung and Choi [5] derived the second-order BEM in which the normal derivatives are defined separately on each adjacent element, and the time interval is determined at each time step by the minimum ratio of the distance between two adjacent grid points to the grid velocity.

Furukawa et al. [6] applied a convolution quadrature method (CQM) to overcome unstable numerical solutions for a small time increment in a conventional time-domain BEM. The statistical properties of sound radiation moving into an unbounded space from vibrating plates have been solved by Choi et al. [7], with a circular plate assumption introduced to simplify the integral expressions. Acoustic pressure radiating from the moving sources governed by linearized Euler equations has been considered as the input in high-order finite-difference schemes and in a high-order Runge-Kutta algorithm for the time-integration by Dragna and BlancBenon [8]. Dragna and Blanc-Benon [9] represented sound radiation by a line source moving at a uniform speed parallel to an impedance plane in the time domain. Dong et al. [10] utilized a coordinate transformation coupled with a cell subdivision technique to solve the singular problem. 


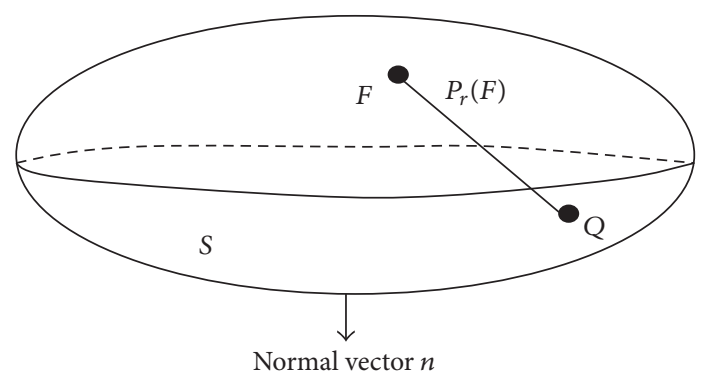

FIgURe 1: A body of arbitrary shape in domain.

According to Rizos and Zhou [11], the impulse response technique can be applied in the time-domain BEM. The solution of the boundary integral equation is presented by the B-spline time modulation of the excitation. Hackbusch et al. [12] employed the convolution quadrature for discretization in time and the Galerkin boundary element method for space discretization. Jang and Ih [13] filtered the troublesome wave vectors at each time step in the time-domain boundary method to stabilize the time step progression. Zheng et al. [14] used the time-domain equivalent source method (TESM) to calculate the radiated waves. Liu and Maury [15] introduced the Acoustic Radiation Mode (ARM) and rewrote the sound pressure at each element of the observation surface as the acoustic impedance matrix multiplied by the normal velocity vector in the frequency domain. Zhou et al. [16] proposed an adaptive background cell-based domain integration method to reduce time consumption during the computing of the boundary integral equation. Isakari et al. [17] converted the eigenvalue problems into nonlinear eigenvalue problems.

This paper derives an Advance-Tracing Boundary Element Method in the time domain to calculate the waves radiated from an arbitrary obstacle accelerating in any direction. The BIE derived herein is the same as the classical retarded potential integral equation. However, a different discretization is processed in the time domain, and a numerical scheme relative to the quarter time period for iterations is proposed. Moreover, this paper emphasizes the discretized formulations based on the BIE instead of the BIE itself.

In comparison with the published literature, the following improvements and innovations are made in the present method. The boundary integration can be treated easily without applying equivalent sources and B-spline interpolations. Any accelerating obstacles can be treated by the present method without any simplification. It is found that the known Neumann boundary condition is relative to the quarter period of the moving velocity, $T / 4$. The iteration based on the derived formulations is robustly convergent, and it is not necessary to filter any troublesome wave at each time step in the time domain. Therefore, both nonuniform and accelerating speeds, not only uniform speed, can be considered for the obstacle. Robust results are obtained by this Advance-Tracing Boundary Element Method. During the approaching process, the numerical inaccuracy due to time interpolation is minimized by using a Fourier series. A sphere, a cube with sharp edges, and irregular geometry are treated as the computing samples in this paper, and evidence is offered regarding the stability and reliability of the present method.

\section{Theoretical Formulations}

An arbitrarily shaped body is considered, as shown in Figure 1 . The body surface is denoted by $S$, its outward normal by $n$, and the radiated acoustic pressure by $P_{r}$.

By applying Green's second identity and the Sommerfeld radiation condition, a boundary integral equation is defined on $S$ :

$$
\begin{aligned}
& C(F) P_{r}(F) \\
& \quad=\int_{S}\left(P_{r}(Q) \frac{\partial G(F, Q)}{\partial n}-G(F, Q) \frac{\partial P_{r}(Q)}{\partial n}\right) d S .
\end{aligned}
$$

Here, $G(F, Q)$ is Green's function that depends on both locations of a field point, $F$, and a source point, $Q$ :

$$
G(F, Q)=\frac{1}{4 \pi} \frac{e^{-i k r}}{r}
$$

where $r$ is the distance between $F$ and $Q$; and $C(F)$ in (1) is the solid angle at the field point $F$. As the elements on the radiator's surface are smooth, the value of $C(F)$ is 0.5 .

Equation (1) can be expressed in the time domain by the inverse Fourier transform:

$$
\begin{aligned}
C(F) & P_{r}(F, t) \\
= & \int_{-\infty}^{\infty} \int_{S} P_{r}(Q) \cdot \frac{\partial G(F, Q)}{\partial n} \cdot e^{i \omega t} d S d \omega \\
& \quad-\int_{-\infty}^{\infty} \int_{S} G(F, Q) \cdot \frac{\partial P_{r}(Q)}{\partial n} \cdot e^{i \omega t} d S d \omega \\
= & \text { Dipole Term + Source Term. }
\end{aligned}
$$

Because the first term on the right-hand side of (3) is relative to the unit dipole strength, $\partial(1 / r) / \partial n$, it is named the dipole term. Similarly, the second term on the right-hand side of (3) is associated with the unit source strength, $1 / r$; therefore, it is named the source term. 


\section{Advance-Tracing Boundary Element Method}

The dipole term in (3) can be expressed in the time domain as

$$
\begin{gathered}
\frac{1}{4 \pi} \int_{-\infty}^{\infty} \int_{S} P_{r}(Q) \cdot \frac{\partial\left(e^{-i k r} / r\right)}{\partial n} \cdot e^{i \omega t} d S d \omega=\frac{1}{4 \pi} \\
\cdot \int_{-\infty}^{\infty} \int_{S} P_{r}(Q) \cdot e^{i \omega t} \cdot e^{-i k r} \cdot \frac{\partial(1 / r)}{\partial n} d S d \omega+\frac{1}{4 \pi} \\
\cdot \int_{-\infty}^{\infty} \int_{S} P_{r}(Q) \cdot e^{i \omega t} \cdot \frac{1}{r} \cdot \frac{\partial\left(e^{-i k r}\right)}{\partial n} d S d \omega=\frac{1}{4 \pi} \\
\cdot \int_{-\infty}^{\infty} \int_{S} P_{r}(Q) \cdot e^{i \omega(t-r / c)} \cdot \frac{\partial(1 / r)}{\partial n} d S d \omega+\frac{1}{4 \pi} \\
\cdot \int_{-\infty}^{\infty} \int_{S} P_{r}(Q) \cdot e^{i \omega t} \cdot \frac{1}{r} \cdot e^{-i k r} \cdot i k \cdot r^{2}
\end{gathered}
$$$$
\text { Dipole Term }=\sum_{m=1}^{M}\left\{\left[P_{r}\left(Q, t-\frac{r}{c}\right) \cdot\left(\frac{-1}{r^{2}}\right)-\frac{\partial P_{r}(Q, t-r / c)}{\partial t} \cdot \frac{1}{c r}\right] \cdot(\nabla \vec{r} \cdot \vec{n})\right\}_{m} \cdot \frac{\Delta S_{m}}{4 \pi} \text {. }
$$

$$
\begin{aligned}
& \cdot \frac{\partial(1 / r)}{\partial n} d S d \omega=\frac{1}{4 \pi} \int_{-\infty}^{\infty} \int_{S} P_{r}(Q) \cdot e^{i \omega(t-r / c)} \\
& \cdot \frac{\partial(1 / r)}{\partial n} d S d \omega+\frac{1}{4 \pi} \int_{-\infty}^{\infty} \int_{S} P_{r}(Q) \cdot e^{i \omega(t-r / c)} \cdot i k \cdot r \\
& \cdot \frac{\partial(1 / r)}{\partial n} d S d \omega=\frac{1}{4 \pi} \\
& \cdot \int_{S}\left[P_{r}(Q, t-r / c) \cdot \frac{\partial(1 / r)}{\partial n}\right] d S+\frac{1}{4 \pi} \\
& \cdot \int_{S}\left[\frac{\partial P_{r}(Q, t-r / c)}{\partial t} \cdot \frac{r}{c} \cdot \frac{\partial(1 / r)}{\partial n}\right] d S .
\end{aligned}
$$

Thus, the dipole term can be expressed in the time domain as
It is noted that, during the computing process, all the retarded time terms in (5) can be obtained directly from the Fourier series:

$$
\begin{aligned}
& P_{r}\left(Q, t-\frac{r}{c}\right)=A_{0}(Q)+\sum_{n=1}^{\infty} A_{n}(Q) \\
& \cdot \cos \left[n \omega\left(t-\frac{r}{c}\right)\right]+B_{n}(Q) \cdot \sin \left[n \omega\left(t-\frac{r}{c}\right)\right] \\
& \frac{\partial P_{r}(Q, t-r / c)}{\partial t}=n \omega\left\{\sum_{n=1}^{\infty}-A_{n}(Q) \cdot \sin \left[n \omega\left(t-\frac{r}{c}\right)\right]\right. \\
& \left.\quad+B_{n}(Q) \cdot \cos \left[n \omega\left(t-\frac{r}{c}\right)\right]\right\} .
\end{aligned}
$$

Equations (6) and (7) are used to replace the interpolations for the values of $P_{r}(Q, t-r / c)$ and $\partial P_{r}(Q, t-r / c) / \partial t$ during the iterating process. In the present scheme, the iteration is based on (4). Both $P_{r}(Q, t-r / c)$ and $\partial P_{r}(Q, t-r / c) / \partial t$ are calculated by the Fourier series instead of interpolations and applied on the right-hand side of (4) to update the value of the left-hand side. Because the time interpolations of $P_{r}(Q, t-r / c)$ and $\partial P_{r}(Q, t-r / c) / \partial t$ in the time domain are expressed in close forms, the numerical errors generated by the time interpolations are minimized.

The source term in (3) can be expressed as

$$
\begin{aligned}
\text { Source Term } & =-\int_{-\infty}^{\infty} \int_{S} G(F, Q) \cdot \frac{\partial P_{r}(Q)}{\partial n} \cdot e^{i \omega t} d S d \omega \\
& =\frac{-1}{4 \pi} \int_{S}\left[\left(\frac{1}{r}\right) \cdot \frac{\partial P_{r}(Q, t-r / c)}{\partial n}\right] d S .
\end{aligned}
$$

In (8), the radiated pressure, $P_{r}$, can be presented by the velocity potential, $\phi\left(t_{s}\right)$ :

$$
P_{r}\left(Q, t_{s}\right)=-\rho \frac{\partial \phi\left(Q, t_{s}\right)}{\partial t_{s}}
$$

Equation (9) was derived by the linearized continuity equation and linear inviscid force equation in Kinsler et al. [18] and is usually used to represent acoustic pressure. In order to reformulate the term, $\partial P_{r}\left(Q, t_{s}\right) / \partial n$, as a time-harmonic Neumann boundary condition relative to the motion velocity, (9) is imposed.

We note that $\rho$ is the mean density of the fluid. The velocity potential can be formed by the Fourier series as

$$
\begin{aligned}
\phi\left(Q, t_{s}\right) & =C_{0}(Q)+\sum_{J=1}^{\infty} C_{J}(Q) \cos \left[J \omega t_{s}-\varsigma_{J}(Q)\right] \\
& =C_{0}(Q)+\sum_{J=1}^{\infty} \phi_{J}\left(Q, t_{s}\right) .
\end{aligned}
$$

Equation (9) can thus be rewritten as

$$
\begin{aligned}
P_{r}\left(Q, t_{s}\right)= & \rho \sum_{J=1}^{\infty} J \cdot \omega \cdot C_{J}(Q) \sin \left[J \omega t_{s}-\varsigma_{J}(Q)\right] \\
= & \rho \sum_{J=1}^{\infty} J \cdot \omega \\
& \cdot C_{J}(Q) \cos \left[J \omega t_{s}-\varsigma_{J}(Q)-\frac{\pi}{2}\right] \\
= & \rho \sum_{J=1}^{\infty} J \cdot \omega \cdot C_{J}(Q) \cos \left[J \omega t_{s}-\xi_{J}(Q)\right] \\
= & \rho \sum_{J=1}^{\infty} J \cdot \omega \cdot \phi_{J}^{*}\left(Q, t_{s}\right) .
\end{aligned}
$$


Applying (10) and (11) into the pressure differential term, $\partial P_{r}(Q, t-r / c) / \partial n$, in (8), the pressure differential term can now be reformed as

$$
\begin{aligned}
\frac{\partial P_{r}\left(Q, t_{s}\right)}{\partial n} & =\rho \cdot \omega \sum_{J=1}^{\infty} J \frac{\partial \phi_{J}^{*}\left(Q, t_{s}\right)}{\partial n} \\
& =\rho \cdot \omega \sum_{J=1}^{\infty} J \cdot V_{J}^{n *}\left(Q, t_{s}\right) \\
& =\rho \cdot \omega \sum_{J=1}^{\infty} J \frac{\partial \phi_{J}\left(Q, t_{s}-(\pi / 2) \cdot(1 / J \omega)\right)}{\partial n} \\
& =\rho \cdot \omega \sum_{J=1}^{\infty} J \frac{\partial \phi_{J}\left(Q, t_{s}-(T / 4) \cdot(1 / J)\right)}{\partial n} \\
& =\rho \cdot \omega \sum_{J=1}^{\infty} J V_{J}^{n}\left(Q, t_{s}-\frac{T}{4} \cdot \frac{1}{J}\right) .
\end{aligned}
$$

Here, $T$ is the period of the moving velocity. It is shown from (12) that the term, $\partial P_{r}\left(Q, t_{s}\right) / \partial n$, can be represented by the normal velocity, $V_{J}^{n}$, is harmonic on each element, and is associated with the quarter period of the moving velocity, $T / 4$.

The normal velocity harmonic on the $m$ th element, $\left[V_{J}^{n}\right]_{m}$, can be presented by the Fourier series:

$$
\begin{aligned}
& \vec{V}\left(Q, t_{s}\right) \cdot \vec{n}_{m} \\
& =D_{0}^{m}(Q)+\sum_{J=1}^{\infty} D_{J}^{m}(Q) \cdot \cos \left[J \omega t_{s}-\beta_{J}^{m}(Q)\right] \\
& \quad=D_{0}(Q)+\sum_{J=1}^{\infty}\left[V_{J}^{n}\left(Q, t_{s}\right)\right]_{m} \\
& {\left[V_{J}^{n}\left(Q, t_{s}-\frac{T}{4} \cdot \frac{1}{J}\right)\right]_{m}} \\
& \quad=D_{J}^{m}(Q) \cdot \cos \left[J \omega\left(t_{s}-\frac{T}{4} \cdot \frac{1}{J}\right)-\beta_{J}^{m}(Q)\right],
\end{aligned}
$$

where $\vec{V}$ is the moving speed of the obstacle. Finally, the source term in (3) can be derived as

$$
\begin{aligned}
& \text { Source Term }=-\rho \cdot \omega \sum_{m=1}^{M} \sum_{J=1}^{\infty} J \cdot D_{J}^{m}(Q) \\
& \cdot \cos \left[J \omega\left(t_{s}-\frac{T}{4} \cdot \frac{1}{J}\right)-\beta_{J}^{m}(Q)\right] \cdot \frac{1}{r_{m}} \cdot \frac{\Delta S_{m}}{4 \pi} .
\end{aligned}
$$

According to (15), the known Neumann boundary condition is relative to the quarter period of the moving velocity, $T / 4$. As the radiated noise at time $t, P_{r}(F, t)$, is computed, the known boundary condition shown in (15) is imposed and is associated with the time $t_{s}-(T / 4) \cdot(1 / J)$.

$$
t_{s}-\frac{T}{4} \cdot \frac{1}{J}=t-\frac{r}{c}-\frac{T}{4} \cdot \frac{1}{J} .
$$

Equation (16) indicates that the radiated noise at time $t$, $P_{r}(F, t)$, should be traced forwardly by the retarded time, $r / c$, and the quarter period of the moving velocity, $T / 4$.

\section{Step Approach Process}

A step approach process is applied to solve the radiated waves of the obstacle moving with acceleration. The step approach method is based on (3). The source term in (15) is a boundary condition and is known. An initial radiated pressure, $P_{r}$, on the body equal to zero is first prescribed. The radiated pressure field on the body on the left-hand side of (3) can then be updated explicitly by calculating the dipole term according to $(5) \sim(7)$. The time step is repeated over the longest period $\left(T=1 / f_{\text {MIN }}\right)$ of the multifrequency moving speed of the obstacle; this process is repeated until the proper convergence is achieved. The time marching is divided into $n$ time steps $($ ISTEP $=n$ ) within the longest period.

The procedure of the present scheme is indicated by an example, shown in Figure 2. In Figure 2, there is a total of three elements $(1 \sim 3)$ on the obstacle, and the time marching is divided into 3 time steps within the longest period. For the first loop, the initial radiated pressure of every element at each time step is set as zero. At the first time step, the radiated pressure of every element is updated according to the right-hand side of (3). The updated values are P111, P211, and P311. Similarly, the updated values of each element at the 3rd time step are P131, P231, and P331 individually. As the first loop is finished, the radiated pressures on all elements are updated. The updated values of the first loop are then used as the initial values for the second loop, and the approach process is the same as that of the first loop. After several loops, a robust convergence can be achieved, and the corrected radiated pressure on each element is approached within an acceptable numerical error.

\section{Numerical Results}

5.1. A Simple Verification of the Present Method. Figure 3 shows a sphere with radius $(R) 1 \mathrm{~m}$ in an infinite air domain. The center of the sphere is at $(0,0,0)$. The mean density of the air is $1.21 \mathrm{~kg} / \mathrm{m}^{3}$, and the sound speed is $343 \mathrm{~m} / \mathrm{s}$. The sphere has a monofrequency motion. The definitions of motion speed for each element shown in (12) are

$$
\begin{aligned}
D_{1}^{m}(Q) & =1(\mathrm{~m} / \mathrm{s}) ; \\
\beta_{1}^{m}(Q) & =0 ; \\
\omega & =343(\mathrm{rad} / \mathrm{s}) .
\end{aligned}
$$

A total of 800 elements are used to model the sphere, and one iteration loop is divided into 20 time steps. Figure 4 indicates that the present Advance-Tracing Boundary Element Method easily achieves a robust convergence. The maximum difference of radiated pressure on any element between the $(n+1)$ th and $n$th loops $\left(P_{r, n+1}-P_{r, n}\right)$ is less than $10^{-3}(\mathrm{~Pa})$ 


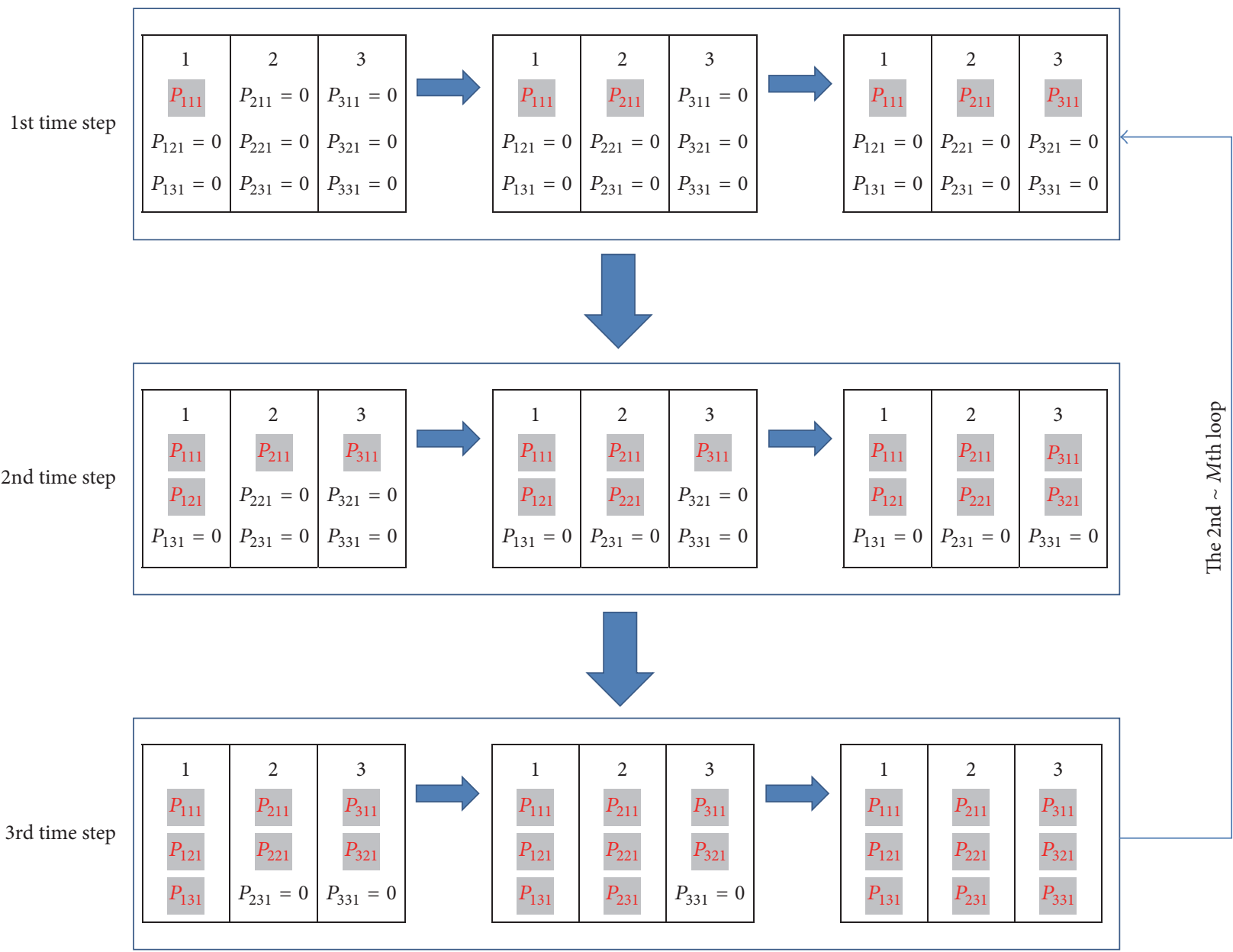

$P_{i j k}$ ( $i$ element, $j$ time step, $k$ loop)

$P_{i j k}$ : the updated value is marked by the grey shade

Figure 2: Flow chart of the numerical implementation (3 elements and 3 time steps).

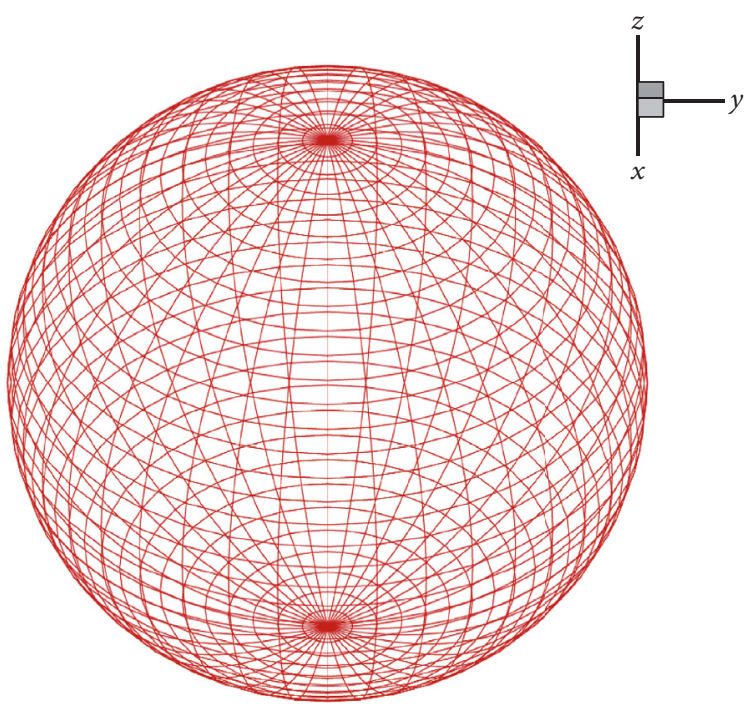

FIGURE 3: Sphere in the infinite air domain for the case in Section 5.1. 


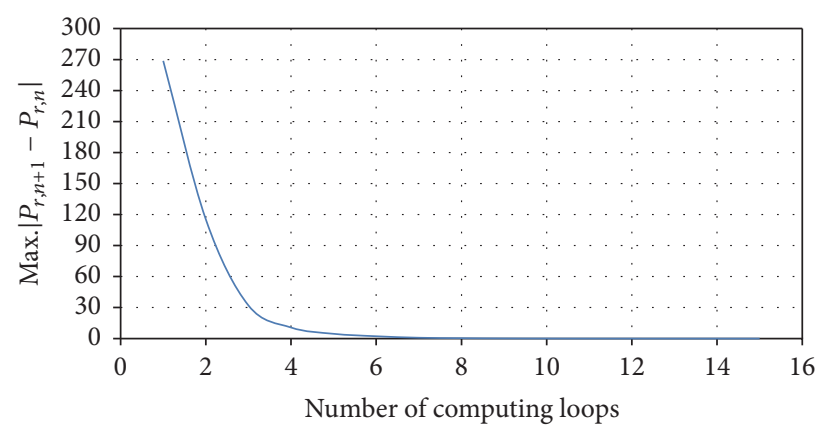

FIgURE 4: Convergence history for the case in Section 5.1.

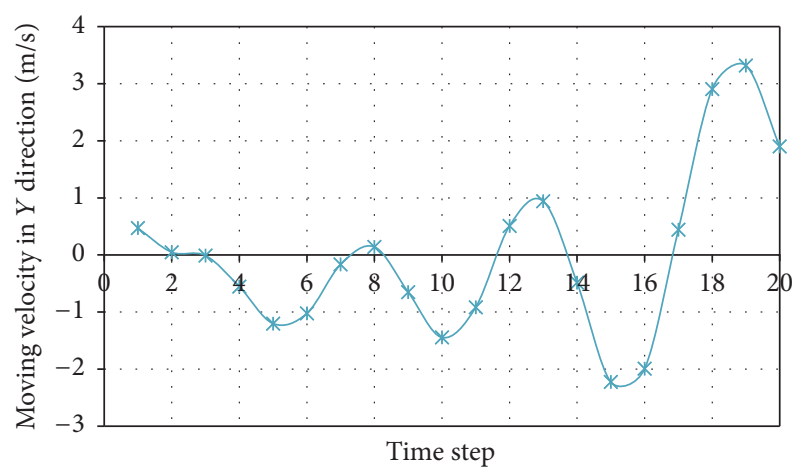

FIGURE 5: Moving velocity of the immersed obstacle for the case in Section 5.2.

after the eighth loop, and the convergent value of the radiated pressure is $291.2 \mathrm{~Pa}$. The analytical solution for this case is

$$
P_{r}=\left(\frac{R^{2}}{r}\right) \frac{i \rho \omega}{1+i k R} e^{-i k(r-R)} .
$$

According to (18), the analytical solution for this case is $293.4 \mathrm{~Pa}$. The error between the numerical and analytical solutions is about $0.75 \%$. This case shows that the numerical solution compares well with the analytical one and that the present method is both stable and reliable.

5.2. Sphere Accelerating Randomly in a Y Direction. The same sphere shown in Figure 3 is also discussed here. The sphere accelerates randomly in a $Y$ direction in an infinite sea water domain. The mean density of the air is $1025 \mathrm{~kg} / \mathrm{m}^{3}$, and the sound speed is $1500 \mathrm{~m} / \mathrm{s}$. Figure 5 illustrates the moving velocity of the immersed obstacle in a $Y$ direction. The time step over the longest period of the moving velocity is 20 (i.e., $\Delta t=0.01 \mathrm{~s}$ ), and the number of elements on the obstacle is 800 . The radiated pressure is calculated by the present method, and the convergence history, as shown in Figure 6, indicates that the present procedure is robustly convergent.

Figure 7 shows the plots for the first-order radiated pressures. Because the acceleration of the immersed sphere is in the $Y$ direction, the source term derived in (14) is larger

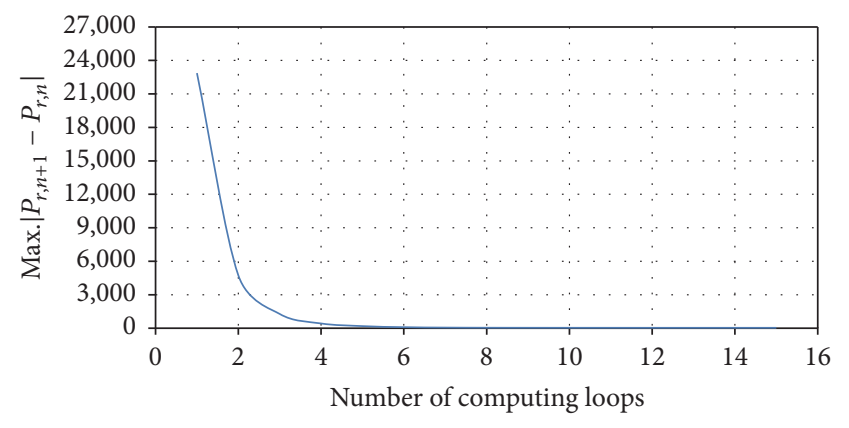

FIgURE 6: Convergence history for the case in Section 5.2.

for those elements close to $|Y|=1 \mathrm{~m}$ due to the remarkable quantity of normal projection in (12). Thus, the strongest radiated pressures appear on the elements close to $|Y|=1 \mathrm{~m}$. As the elements get nearer to the $X-Z$ plane, the normal projection in (12) is nearly zero, and the source term derived in (14) is very small. Reasonably, the weakest radiated pressures appear on the elements near the $X-Z$ plane. The contours in Figure 7 are symmetric relative to the $X-Z$ plane, due to the movement in the $Y$ direction and the symmetry of the discussed obstacle.

5.3. Cubic Shape Accelerating Randomly in a Y Direction. In this case, the radiating obstacle is a cube with sharp edges immersed in sea water, as shown in Figure 8. The side length of the cube is $2 \mathrm{~m}$, and the center is at $(0,0,0)$. The characteristics of the sea water and the moving velocity of the cube are the same as those for Section 5.2. There are 1176 elements on the obstacle. The convergent history is shown in Figure 9, and robust convergence is easily achieved. It is also evident in Figure 9 that the present method is suitable for complex geometry in which sharp edges are included.

The first-order radiated pressures are contoured in Figure 10. On the planes whose normal vectors are parallel to the $Y$-direction motion, the radiated pressures are the greatest due to the source term derived in (15). For the other planes whose normal vectors are vertical to the $Y$-direction motion, the radiated pressures are relatively small. The radiated pressures are symmetric to the $X-Z$ plane, because 

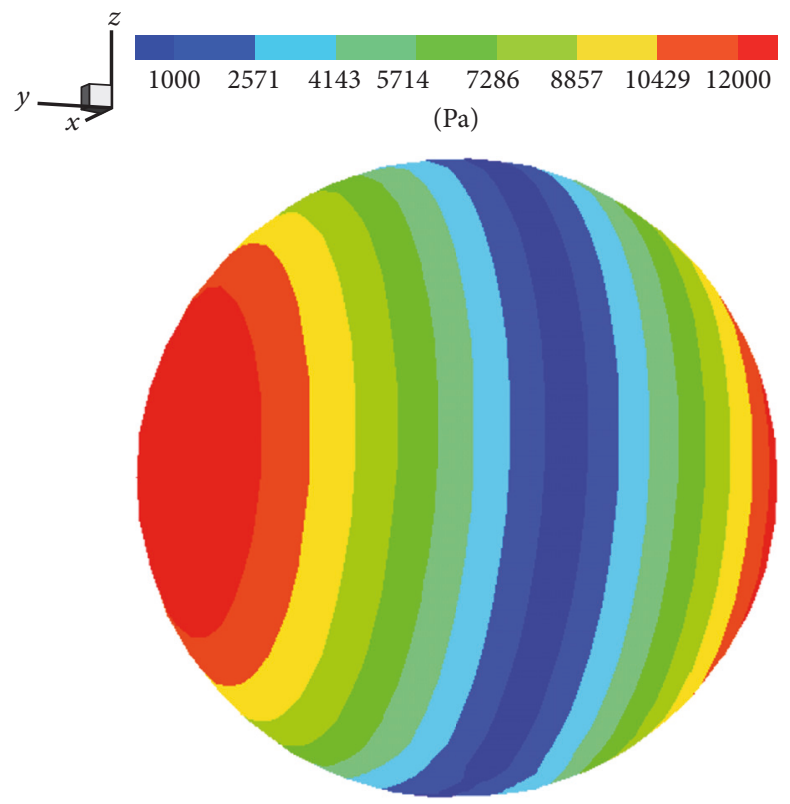

FIGURE 7: The first-order radiated pressure for the case in Section 5.2.

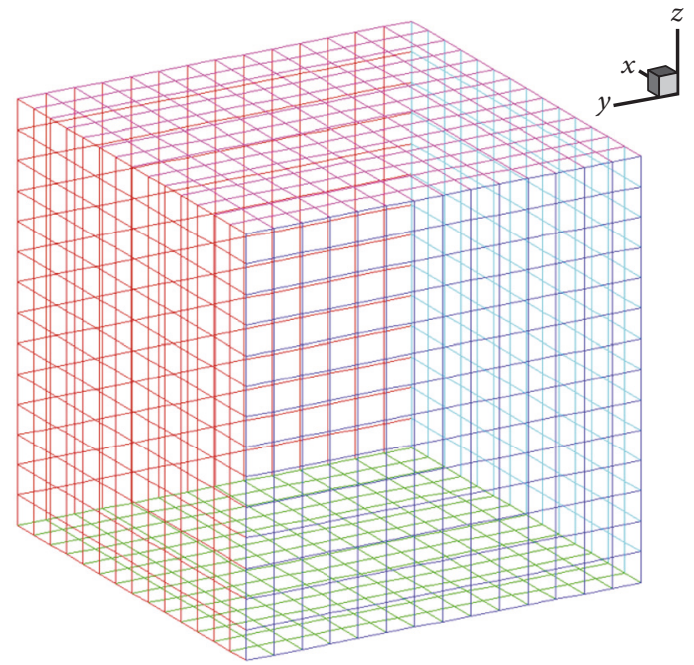

FIGURE 8: Cubic in the infinite sea water domain for the case in Section 5.3.

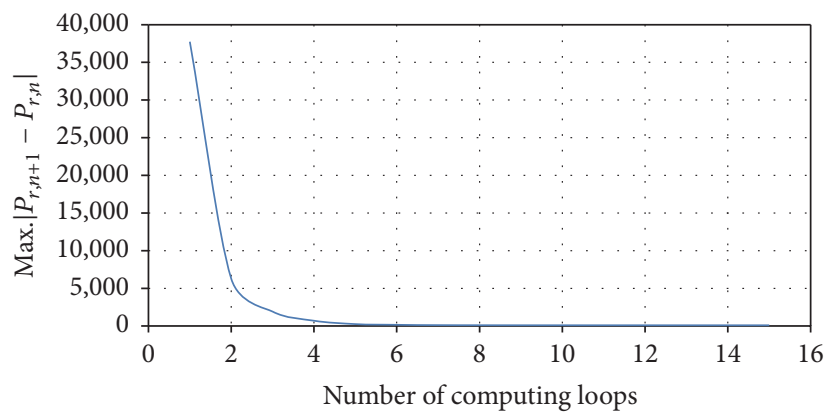

FIGURE 9: Convergence history for the case in Section 5.3. 


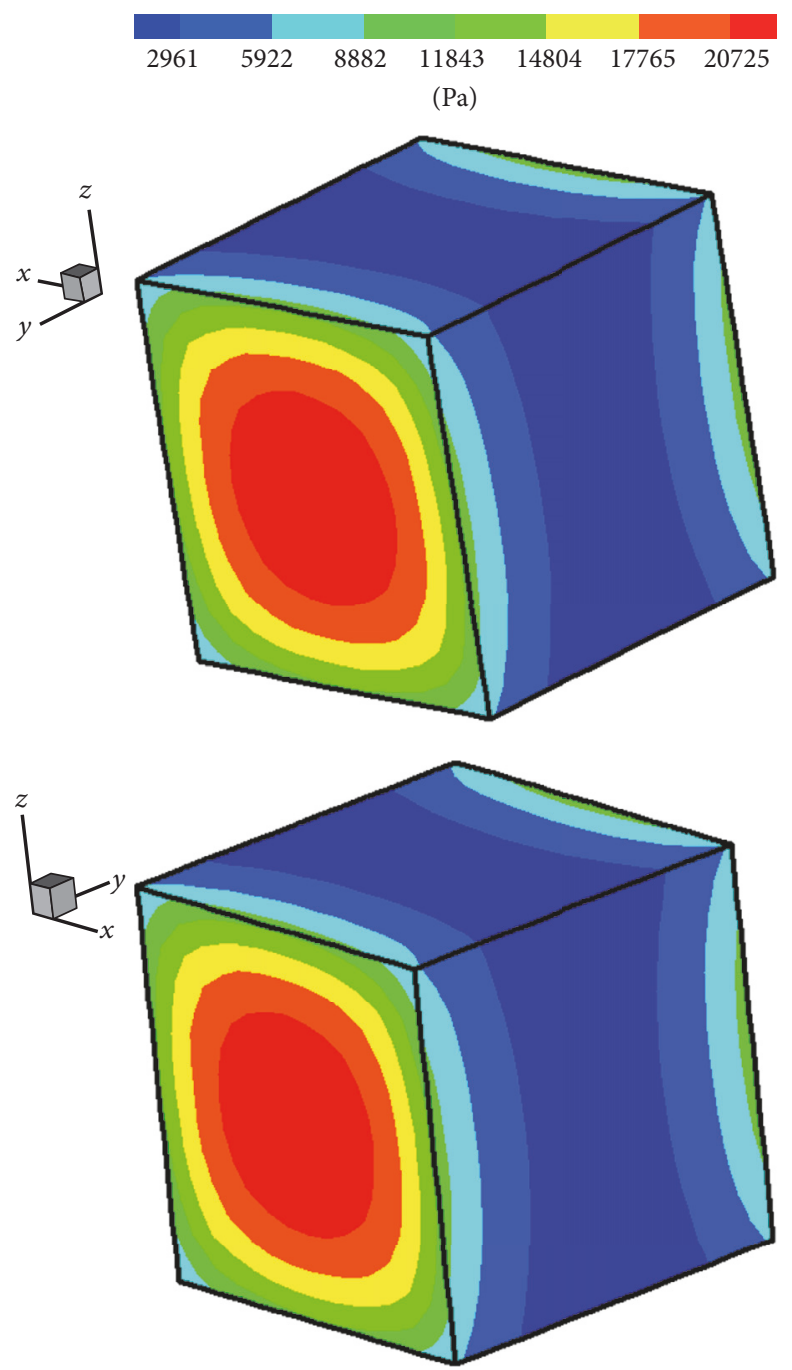

FIgURE 10: The first-order radiated pressure for the case in Section 5.3.

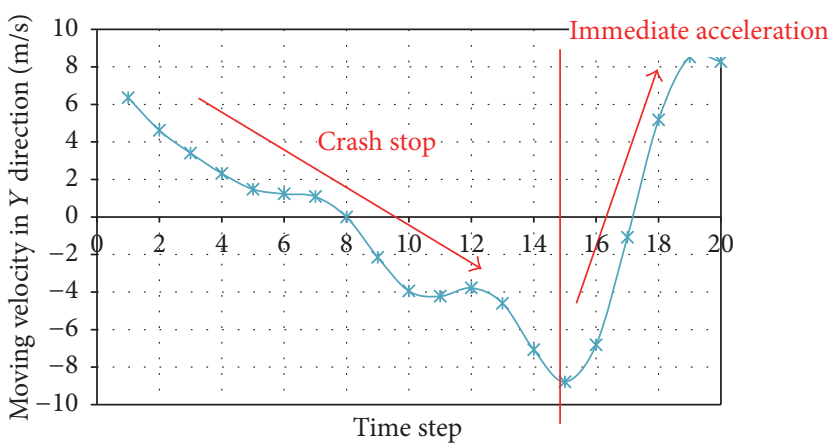

FIGURE 11: Moving velocity of the immersed obstacle for the case in Section 5.4.

of the $Y$-direction motion and symmetry of the discussed obstacle.

5.4. Cubic Shape Moving with a Crash Stop and Immediate Acceleration in a Y Direction. The same obstacle discussed in the case of Section 5.3 is treated as the computing sample here, but the moving velocity of a cube in a $Y$ direction includes a crash stop and immediate acceleration. Figure 11 presents the moving velocity of the cube. The robust convergence is recorded in Figure 12, and it is found that 


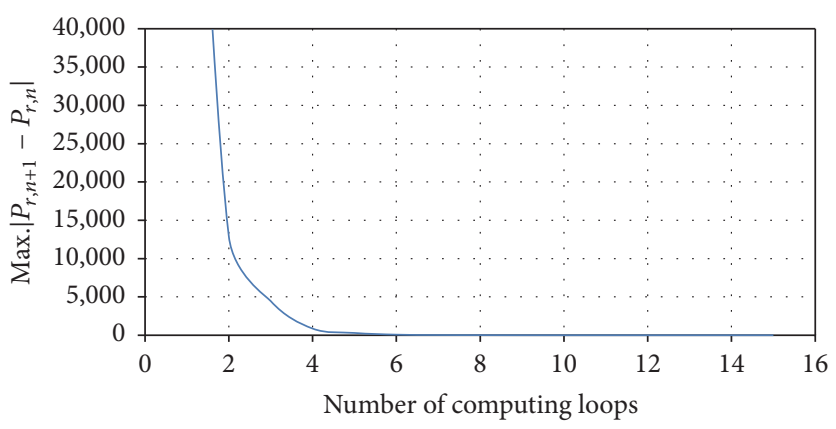

FIGURE 12: Convergence history for the case in Section 5.4.

$\begin{array}{lllllll}17764 & 35529 & 53293 & 71058 & 88822 & 106589 & 124351\end{array}$

$(\mathrm{Pa})$
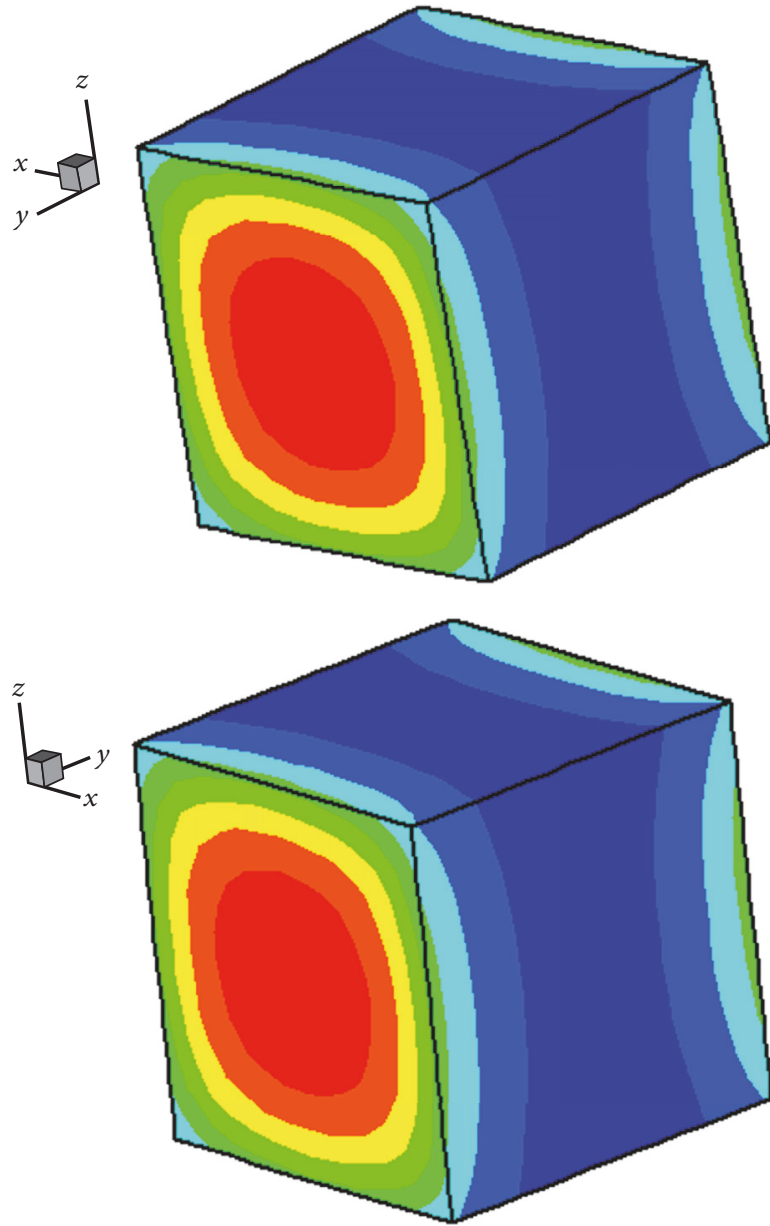

Figure 13: The first-order radiated pressure for the case in Section 5.4.

the present method can be applied in complex geometry moving with a velocity that varies rapidly. The first-order radiated pressures are plotted in Figure 13, whereby their distribution is reasonably similar to that in Figure 10. Because the acceleration for this case varies more immediately than that for the case of Section 5.3, the amplitudes shown in Figure 13 are distinctly stronger than those shown in Figure 10. 


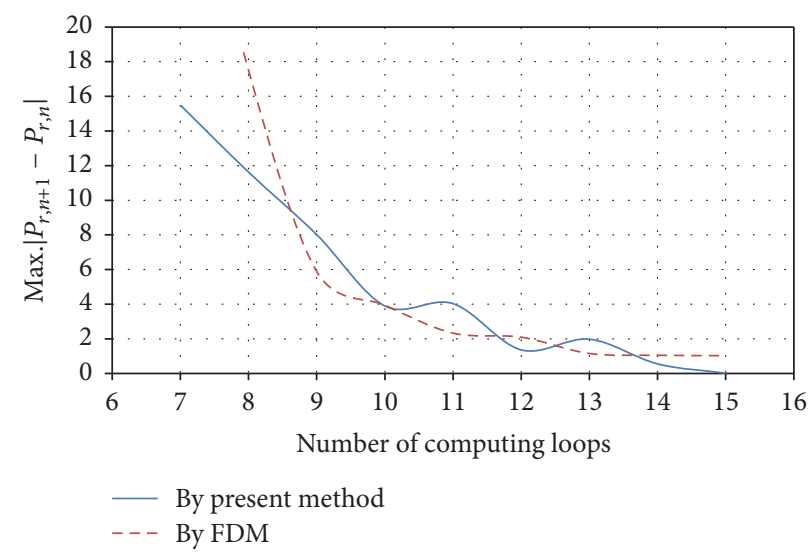

FIGURE 14: Convergence histories for the case in Section 5.4 by the present method and FDM.

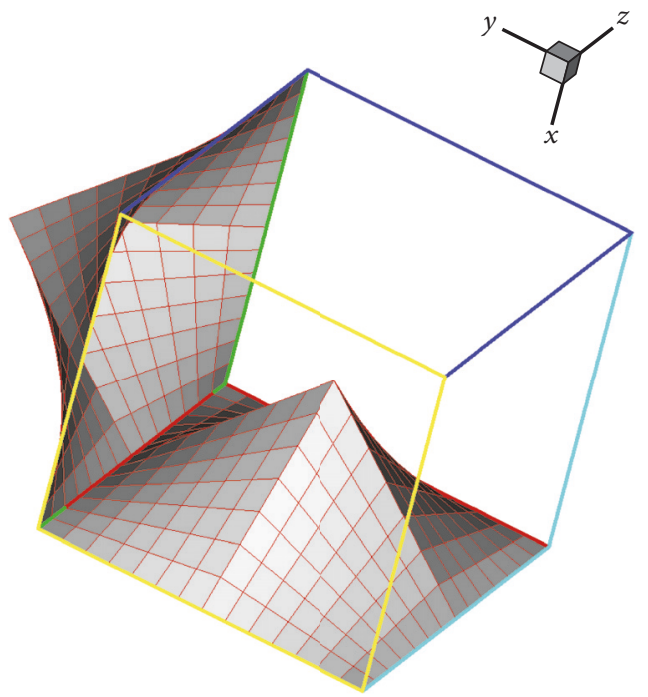

FIGURE 15: An irregular radiator in the infinite sea water domain for the case in Section 5.5.

As (7) is replaced by finite-difference method (FDM), (7) can be expressed as

$$
\begin{aligned}
& \frac{\partial P_{r}\left(\mathrm{Q}, t_{S}\right)}{\partial t} \\
& =\frac{-3 P_{r}\left(\mathrm{Q}, t_{S}\right)+4 P_{r}\left(\mathrm{Q}, t_{S}+\Delta t\right)-P_{r}\left(\mathrm{Q}, t_{S}+2 \Delta t\right)}{2 \cdot \Delta t} .
\end{aligned}
$$

By computing the same case, Section 5.4, Figure 14 shows that the convergence could be obtained more quickly by the present method than by FDM.

5.5. Irregular Geometries. Another geometry shown in Figure 15 is considered to test the proposed method. The geometry is the same cubic as in the case of Section 5.4, but two planes, 1 and 2, are remarkably irregular. The computing conditions are the same as those in the case of Section 5.4. The iterating history plotted in Figure 16 is robust. The first-order radiated pressures are contoured in Figure 17. For planes 1 and 2, the radiated pressures are symmetric to the $X-Y$

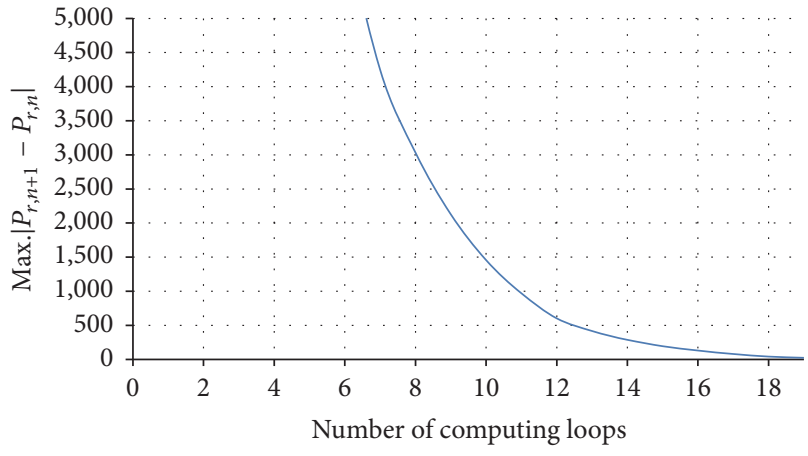

FIGURE 16: Convergence history for the case in Section 5.5.

plane, because of the $Y$-direction motion and the geometric symmetry to the $X-Y$ plane for the discussed obstacle. The normal projection of the motion velocity on plane 1 is larger than that on plane 2; thus, the pressure amplitude on plane 1 is stronger than that on plane 2 . 


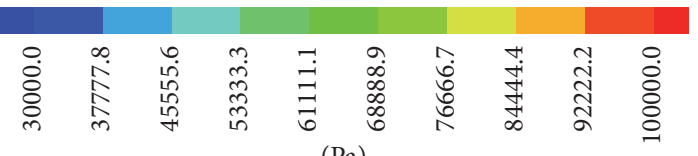

$(\mathrm{Pa})$
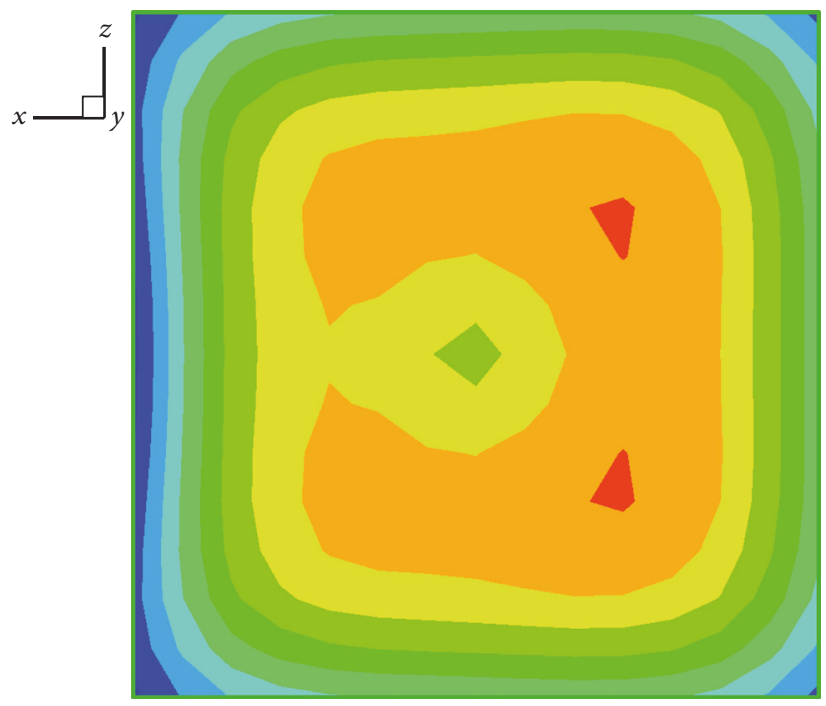

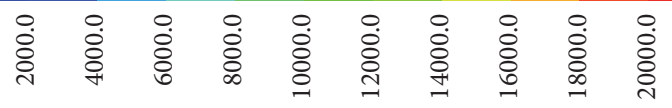

$(\mathrm{Pa})$
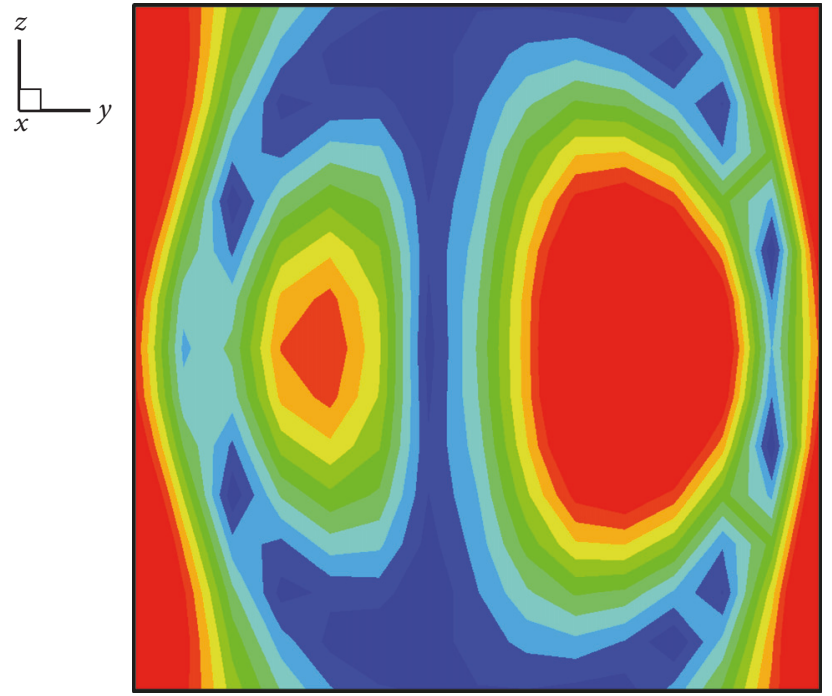

FIGURE 17: The first-order radiated pressure for the case in Section 5.5.

\section{Conclusions}

This study develops an Advance-Tracing Boundary Element Method in the time domain to calculate the waves radiating from an immersed obstacle accelerating randomly. The scheme is derived in the time domain, and the moving velocity is treated as the known boundary condition relative to the advance-tracing time, which is equal to the quarter period of the moving velocity. The moving velocity could be multifrequency, and the immersed obstacle could be complexly shaped with irregular geometry.

The radiated pressure from a sphere moving with constant velocity amplitude in single frequency is shown to be in good agreement when compared with the analytical solution. The multifrequency moving velocity (i.e., random acceleration) is then imposed upon the same sphere. It is shown that robust convergence can be obtained by means of the present method, 
and strong radiations appear on the elements for where the normal vector is parallel to the moving velocity.

This paper also tests another obstacle, a cube with sharp edges. Robust convergence is obtained for the same multifrequency moving velocity discussed in the sphere sample. When the multifrequency moving velocity is modified to include a crash stop and immediate acceleration, and when the plane is shaped irregularly, a convergent history can also be easily achieved. It is found that the radiated pressures increase due to severe variations in moving velocity. Due to the larger boundary condition values, the radiated pressures are more remarkable on those elements whose normal vectors are more parallel to the moving velocity. The present method is clearly very suitable for arbitrary obstacles with random acceleration.

\section{Conflicts of Interest}

The author declares that they have no conflicts of interest.

\section{References}

[1] L. Rayleigh, Theory of Sound, Dover, New York, NY, USA, 1945.

[2] W. Kropp and P. U. Svensson, "Application of the time domain formulation of the method of equivalent sources to radiation and scattering problems. Acta Acoustica," Acta Acoustica, vol. 81, pp. 528-543, 1995.

[3] M. Ochmann, "Source simulation technique for acoustic radiation problems," Acustica, vol. 81, no. 6, pp. 512-527, 1995.

[4] T. W. Wu, "A direct boundary element method for acoustic radiation and scattering from mixed regular and thin bodies," Journal of the Acoustical Society of America, vol. 97, no. 1, pp. 84-91, 1995.

[5] H. G. Sung and H. S. Choi, "Implicit formulation with the boundary element method for nonlinear radiation of water waves," Engineering Analysis with Boundary Elements, vol. 34, no. 5, pp. 511-529, 2010.

[6] A. Furukawa, T. Saitoh, and S. Hirose, "Convolution quadrature time-domain boundary element method for 2-D and 3-D Elastodynamic analyses in general anisotropic elastic solids," Engineering Analysis with Boundary Elements, vol. 39, pp. 6474, 2014.

[7] W. Choi, J. Woodhouse, and R. S. Langley, "Sound radiation from a vibrating plate with uncertainty," Journal of Sound and Vibration, vol. 333, no. 17, pp. 3966-3980, 2014.

[8] D. Dragna and P. Blanc-Benon, "Towards realistic simulations of sound radiation by moving sources in outdoor environments," International Journal of Aeroacoustics, vol. 13, no. 5-6, pp. 405-426, 2014.

[9] D. Dragna and P. Blanc-Benon, "Sound radiation by a moving line source above an impedance plane with frequencydependent properties," Journal of Sound and Vibration, vol. 349, pp. 259-275, 2015.

[10] Y. Dong, C. Lu, Y. Li, J. Zhang, G. Xie, and Y. Zhong, "Accurate numerical evaluation of domain integrals in 3D boundary element method for transient heat conduction problem," Engineering Analysis with Boundary Elements, vol. 60, article no. 3155, pp. 89-94, 2015.

[11] D. C. Rizos and S. Zhou, "An advanced direct time domain BEM for 3-D wave propagation in acoustic media," Journal of Sound and Vibration, vol. 293, no. 1-2, pp. 196-212, 2006.
[12] W. Hackbusch, W. Kress, and S. A. Sauter, "Sparse convolution quadrature for time domain boundary integral formulations of the wave equation," IMA Journal of Numerical Analysis, vol. 29, no. 1, pp. 158-179, 2009.

[13] H. W. Jang and J. G. Ih, "Stabilization of time domain acoustic boundary element method for the interior problem with impedance boundary conditions," The Journal of the Acoustical Society of America, vol. 131, no. 4, pp. 2742-2752, 2012.

[14] X.-Z. Zhang, C.-X. Bi, Y.-B. Zhang, and L. Xu, "Sound source identification and sound radiation modeling in a moving medium using the time-domain equivalent source method," Journal of the Acoustical Society of America, vol. 137, no. 5, pp. 2678-2686, 2015.

[15] Z.-B. Liu and C. Maury, "An improved method for the calculation of Near-Field Acoustic Radiation Modes," Journal of Sound and Vibration, vol. 363, pp. 316-328, 2016.

[16] W. Zhou, Q. Wang, Y. Cheng, and G. Ma, "A fast multipole method accelerated adaptive background cell-based domain integration method for evaluation of domain integrals in 3D boundary element method," Engineering Analysis with Boundary Elements, vol. 67, pp. 1-12, 2016.

[17] H. Isakari, T. Takahashi, and T. Matsumoto, "Periodic band structure calculation by the Sakurai-SUGiura method with a fast direct solver for the boundary element method with the fast multipole representation," Engineering Analysis with Boundary Elements, vol. 68, pp. 42-53, 2016.

[18] L. E. Kinsler, A. R. Frey, A. B. Coppens, and J. V. Sanders, Fundamentals of Acoustics, John Wiley \& Sons, USA, 1982. 


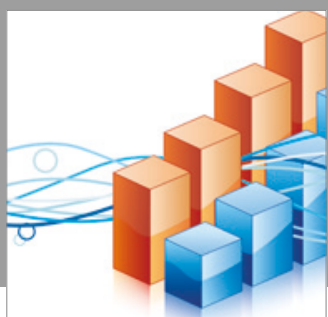

Advances in

Operations Research

vatersals

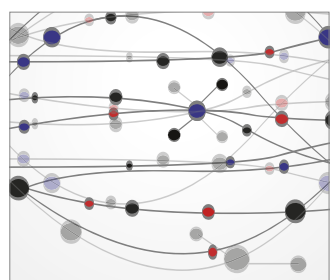

\section{The Scientific} World Journal
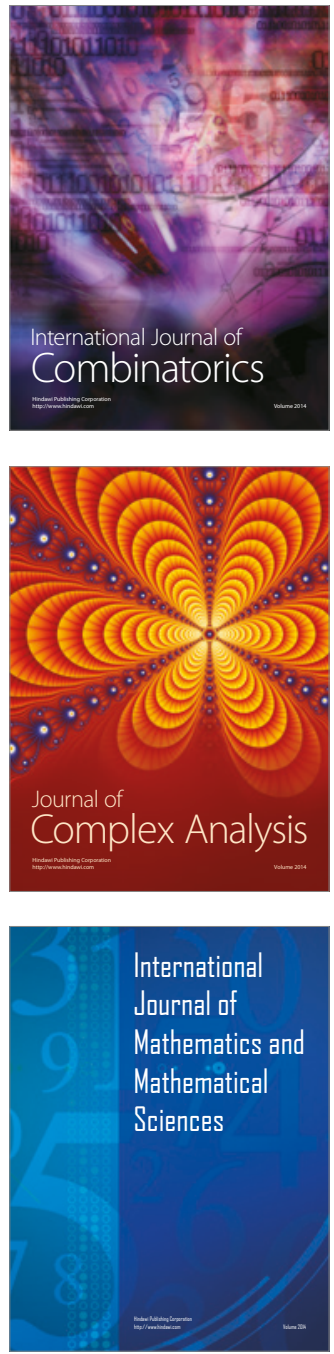
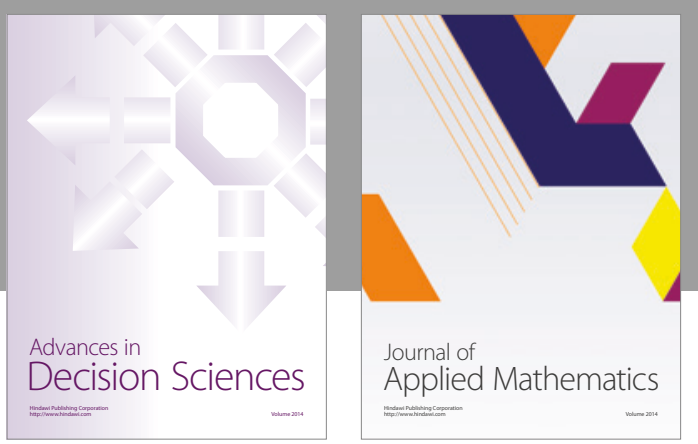

Algebra

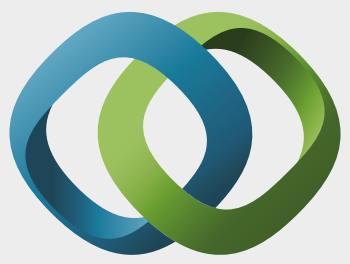

\section{Hindawi}

Submit your manuscripts at

https://www.hindawi.com
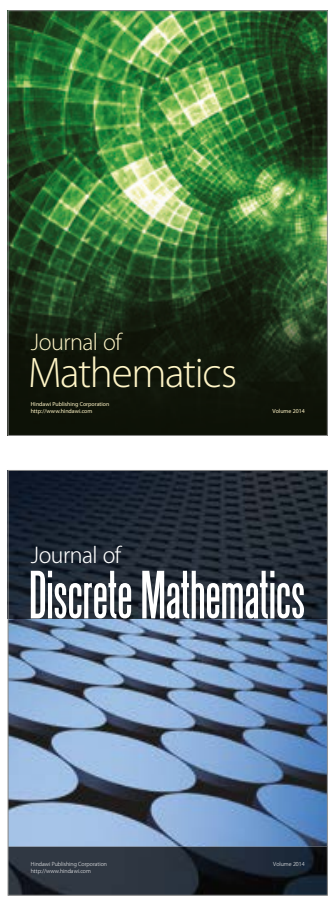

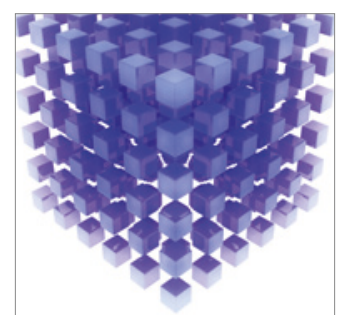

Mathematical Problems in Engineering
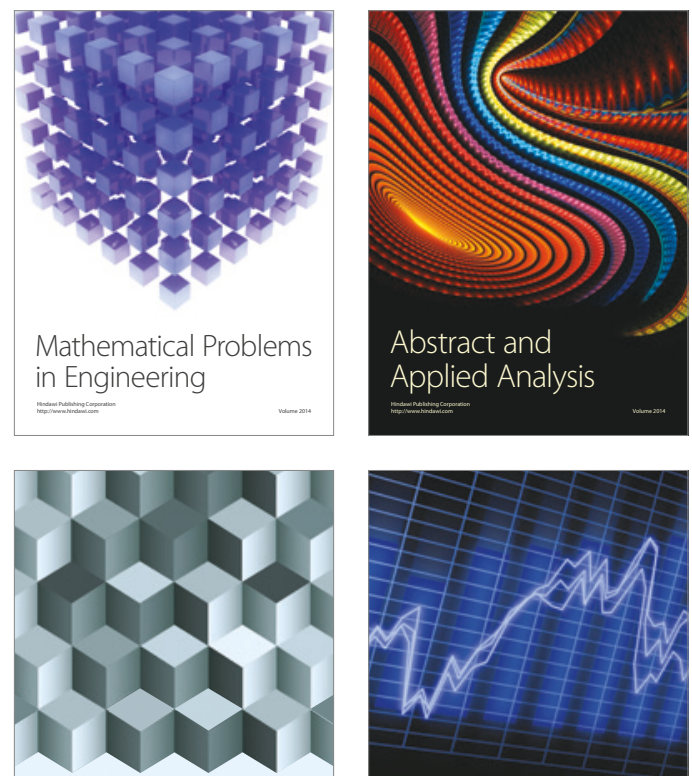

Journal of

Function Spaces

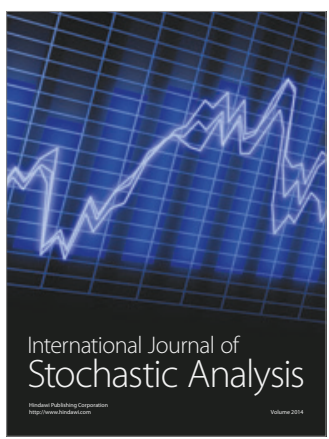

Probability and Statistics
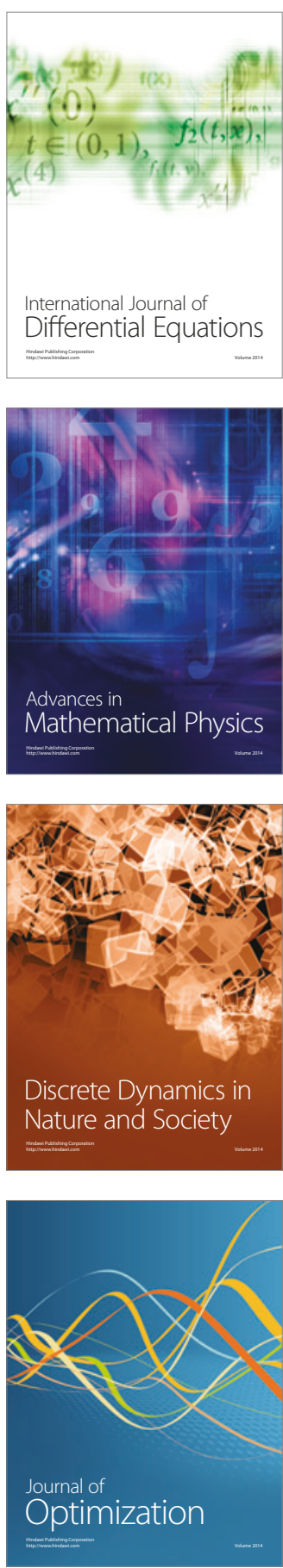